\title{
PROCOLLAGEN PRODUCTION IN FRESH AND CRYOPRESERVED AORTIC VALVE GRAFTS
}

Flavian M. Lupinetti, MD

James M. Kneebone, MS

Mark D. Rekhter, MD

Kelvin G. M. Brockbank, PhD

David Gordon, MD
Long-term durability of aortic valve allografts may be enhanced by cellular capacities for regeneration and repair. To evaluate aortic valve graft production of an important structural protein, rat aortic roots were implanted heterotopically into the abdominal aorta of recipient rats. Grafts were either syngeneic or strongly allogeneic, were implanted either fresh or after cryopreservation, and were left in place 2 to 21 days after implantation. A total of 80 aortic valve grafts and the corresponding native aortic valves were examined. The grafts were retrieved and immunocytochemically stained for the presence of procollagen, a precursor to collagen. Regardless of histocompatibility or preservation, grafts exhibited consistent procollagen presence that equaled or exceeded that seen in the corresponding native valves. Positive procollagen staining was predominantly in the aortic wall. The most prominent staining was near the hinge point of the valve leaflets, with no staining in the free portion of the leaflets. Staining with $\alpha$-actin demonstrated vascular smooth muscle in sites remote from the areas positive for procollagen, which suggests that vascular smooth muscle was not responsible for the procollagen production. These findings indicate that cryopreservation is compatible with persistent fibroblast viability and in vivo protein synthesis by both syngeneic and allogeneic aortic valve grafts. (J Thorac Cardiovasc Surg 1997;113:102-7) ong-term durability of aortic valve allografts may -be enhanced by cellular capacities for regeneration and repair. Persistence of cell viability in allograft valves has been described only sporadically, and maintenance of important cell functions in vivo has not been documented. Questions of preservation techniques are particularly relevant in addressing allograft valve viability and function. It remains a subject of controversy whether cryopreserved heart valves provide results better or

From the Department of Surgery, Division of Cardiothoracic Surgery, the University of Washington, Seattle, Wash.; the Division of Cardiac Surgery, Children's Hospital and Medical Center, Seattle, Wash.; the Department of Pathology, the University of Michigan, Ann Arbor, Mich.; and CryoLife, Inc., Marietta, Ga.

Supported by a Research Endowment Support Grant from the Children's Hospital and Medical Center, Seattle, Wash.

Received for publication March 7, 1996; revisions requested April 8, 1996; revisions received May 6, 1996; accepted for publication July 1, 1996.

Address for reprints: Flavian M. Lupinetti, MD, Division of Cardiac Surgery, CM-03, Children's Hospital and Medical Center, 4800 Sand Point Way NE, Seattle, WA 98105.

Copyright (C) 1997 by Mosby-Year Book, Inc.

$0022-5223 / 97 \$ 5.00+0 \quad \mathbf{1 2 / 1 / 7 6 3 4 8}$ worse than valves subjected to minimal storage methods.

Previous studies from this and other laboratories have examined the properties of endothelial cells in aortic valve grafts. ${ }^{1-5}$ These previous investigations have considered questions of endothelial cell persistence after cryopreservation, replication, and dye exclusion markers of viability. The fibroblast population of the valve graft may ultimately be more important than that of endothelial cells in determining structural stability and function. Fibroblasts have a lower rate of replication than endothelial cells, have no specific cell markers, and are more difficult to subject to some of the quantitative analytic techniques applicable to the endothelium. The present investigation was undertaken to study one aspect of fibroblast viability, specifically the elaboration of procollagen, a precursor molecule to collagen, and therefore an important structural protein. The study was designed to test two hypotheses: first, that cryopreservation of aortic valve grafts is consistent with a capacity for procollagen synthesis similar to that of the native aortic valve and, second, that allografts retain this capacity in a manner similar to that of isografts. 


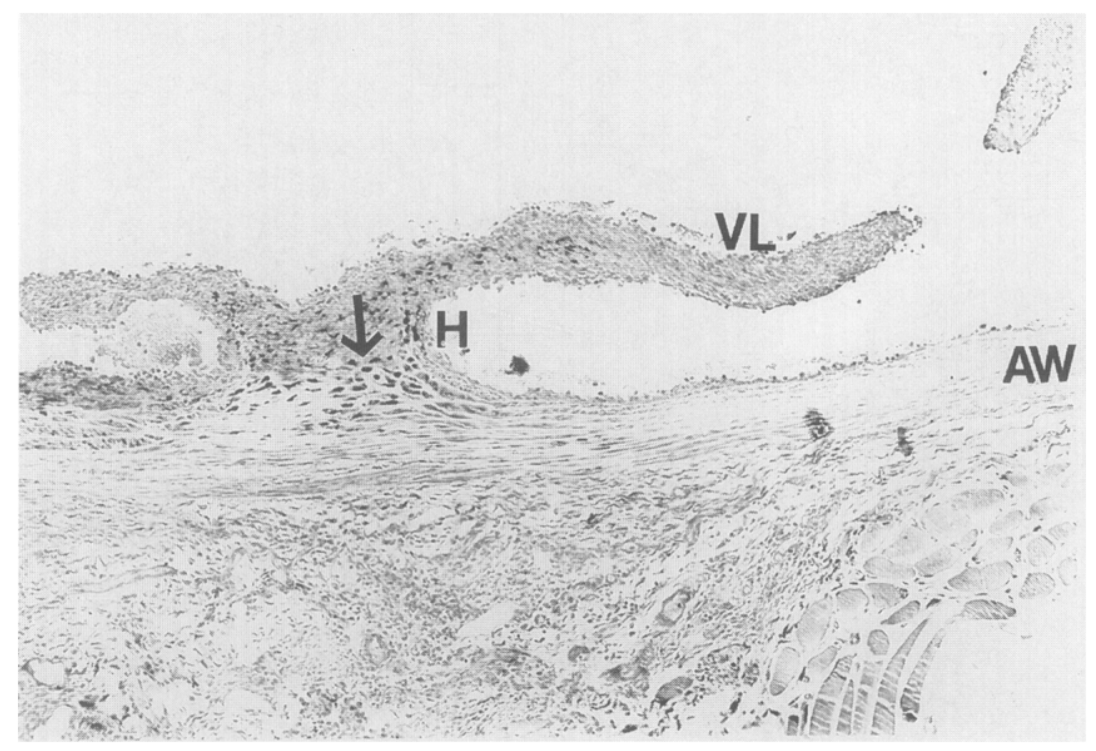

Fig. 1. Aortic valve graft stained for procollagen. Black precipitate (arrow) demonstrates positive staining for procollagen with highest concentration in hinge region $(H)$ of valve and minimal procollagen in aortic wall $(A W)$. There is no procollagen staining in free portion of valve leaflet $(V L)$.

\section{Methods}

Experimental preparation. Heterotopic transplantation of aortic valve grafts was done as described originally by Yankah and colleagues. ${ }^{1}$ Male Lewis rats weighing 100 to $125 \mathrm{gm}$ underwent general anesthesia with intraperitoneal sodium pentobarbital ( $60 \mathrm{mg} / \mathrm{kg}$ body weight). Under sterile conditions, the heart and ascending aorta were excised and rinsed in heparinized $0.9 \%$ saline solution. The aortic valve graft was excised with a $1 \mathrm{~mm}$ cuff of ventricular muscle and a $5 \mathrm{~mm}$ portion of distal artery attached to permit construction of the anastomoses. Some valves were transplanted immediately, whereas others were placed in heparinized saline solution and transported on ice to CryoLife, Inc. (Marietta, Ga.). The grafts underwent antibiotic sterilization, immersion in a graded series of dimethyl sulfoxide concentrations to minimize ice crystal formation during cryopreservation, controlled-rate freezing at a rate of $-1^{\circ} \mathrm{C}$ per minute, and storage in the vapor phase of liquid nitrogen between $-135^{\circ} \mathrm{C}$ and $-196^{\circ} \mathrm{C}$ until use.

At the time of implantation, cryopreserved valves were thawed by immersion in $37^{\circ} \mathrm{C}$ saline solution and rinsed thoroughly before use. Fresh valves were left in heparinized saline solution while the recipient animal was prepared. Aortic valve recipients were male rats weighing 200 to $250 \mathrm{gm}$ of the Lewis (syngeneic) or Brown Norway (strongly allogeneic, RT1 and non-RT1 incompatible) strains. Each recipient underwent sterile laparotomy and dissection of the abdominal aorta under a Nikon SMZ-10 stereoscopic zoom microscope (Garden City, N.Y.) with a usual magnification of $\times 14$. The recipient aorta was occluded proximally and distally with vascular clamps and divided. An end-to-end anastomosis of the distal end of the valve graft to the distal end of the native aorta was done with 8-0 polypropylene suture. A second end-to-end anastomosis between the proximal end of the graft and the proximal end of the native aorta was constructed. None of the leaflets was included in the suture line. Instead, a concerted effort was made to allow the possibility that each leaflet remained mobile so that valve coaptation could occur. The presence or absence of leaflet mobility and coaptation is conjectural, however. The aortic clamps were removed, the abdomen closed, and the animals allowed to recover.

Rats were allowed to survive for $2,7,14$, or 21 days. Thus a total of 80 animals was studied: 20 for each of four durations of implantation, with 5 each receiving fresh isografts, fresh allografts, cryopreserved isografts, and cryopreserved allografts. At the time designated for graft retrieval, the rats received sodium pentobarbital (100 $\mathrm{mg} / \mathrm{kg}$ body weight) administered by intraperitoneal injection. Rats underwent total body perfusion fixation via a femoral artery catheter. Animals were perfused with $40 \mathrm{ml}$ of a fixation solution containing $60 \%$ methanol, $30 \%$ chloroform, and $10 \%$ glacial acetic acid. The perfusate was administered at $120 \mathrm{~mm} \mathrm{Hg}$ after the jugular veins were divided to avoid excessive intravascular pressure. In addition to the 80 animals in the study group, 2 animals died before the designated time for tissue retrieval and 10 that survived were found to have thrombosis of the graft. These animals were excluded from any further analysis. The grafts and native aortic roots were excised and stored for 24 hours in the fixative, dehydrated in graded alcohols and xylene, and embedded in paraffin. Eight-micrometer sections were obtained for histologic staining and analysis.

All animals received humane care in compliance with the "Guide for the Care and Use of Laboratory Animals" published by the National Institutes of Health (NIH Publication No. 85-23, revised 1985).

Histologic analysis. Presence of type I procollagen in native and grafted aortic valves and aortic walls was 


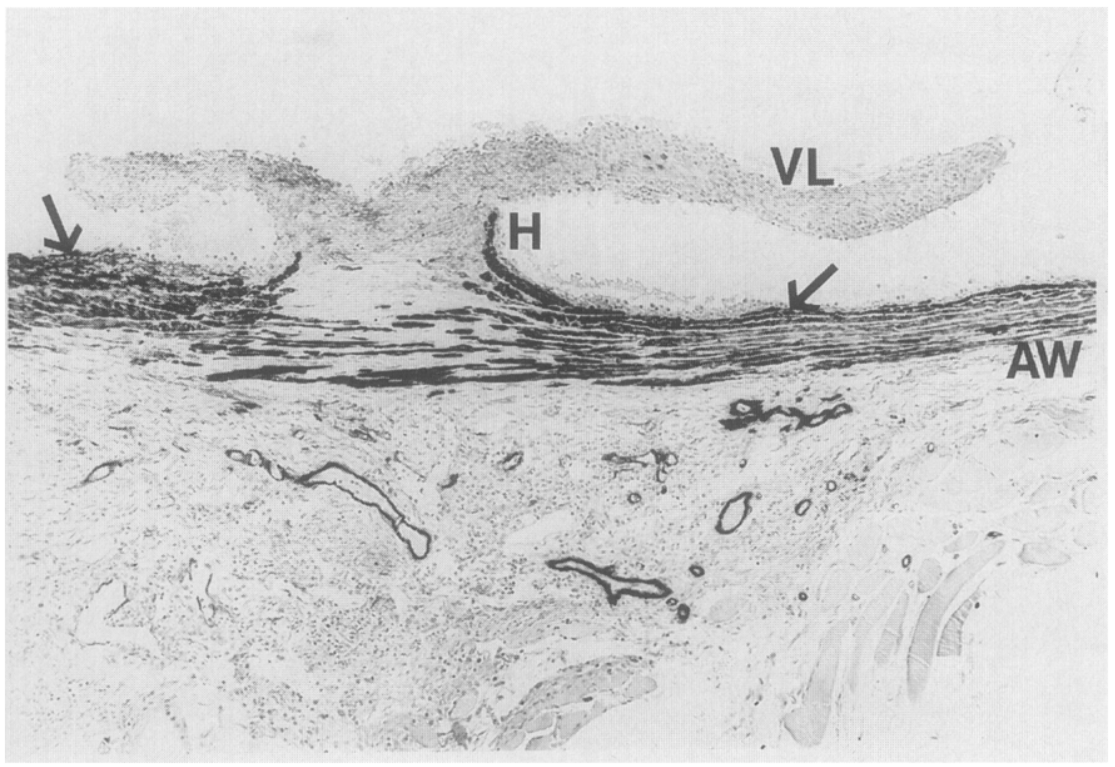

Fig. 2. Serial section of aortic valve graft shown in Fig. 1 stained for $\alpha$-actin. Black precipitate (arrows), demonstrating presence of $\alpha$-actin, is confined to aortic wall $(A W)$ and is remote from hinge region $(H)$, site of most of procollagen staining. No $\alpha$-actin staining was observed in valve leaflet $(V L)$.

determined by the use of immunohistochemical staining as previously described..$^{6-9}$ Mouse monoclonal antibody SPI.D8 was obtained from the Developmental Studies Hybridoma Bank maintained by the Department of Pharmacology and Molecular Sciences, the Johns Hopkins University School of Medicine, and the Department of Biological Sciences, the University of Iowa, under contract N01-HD-6-2915 from the National Institute of Child Health and Human Development. This antibody is directed against type I procollagen aminopeptide. For immunocytochemistry studies, sections were deparaffinized and endogenous peroxidase activity was blocked with $0.3 \% \mathrm{H}_{2} \mathrm{O}_{2}$ for 5 minutes. Undiluted SPI.D8 antibody with $1 \%$ bovine serum albumin in $0.1 \mathrm{~mol} / \mathrm{L}$ phosphatebuffered saline solution was applied for 60 minutes, and the specimen was washed with phosphate-buffered saline solution. The secondary antibody, biotinylated anti-mouse antibody (Vector Laboratories, Burlingame, Calif.) diluted 1:500, was applied for 30 minutes. The specimen was washed and submitted for avidin-biotin amplification with the use of an ABC Elite Kit (Vector Laboratories) for 30 minutes. The specimens were then washed and incubated with $0.1 \% 3^{\prime}, 3^{\prime}$-diaminobenzidine (Sigma, St. Louis, Mo.) and $\mathrm{H}_{2} \mathrm{O}_{2}$ at $37^{\circ} \mathrm{C}$ for 5 to 10 minutes. This produced a brown reaction product. Nuclear counterstaining was done with methyl green for 2 minutes, followed by section dehydration and coverslip mounting.

From the same blocks used to obtain sections for procollagen staining, serial tissue sections were also obtained for staining with an antibody to $\alpha$-actin. The $\alpha$-actin antibody used was a monoclonal mouse antibody to smooth muscle $\alpha$-actin (Boehringer Mannheim, Indianapolis, Ind.). This antibody recognizes smooth muscle cells of all types, including vascular smooth muscle, and reacts with the smooth muscle of many species, including the rat. ${ }^{10}$ Positive staining for $\alpha$-actin in proximity to areas positive for procollagen would suggest that smooth muscle cells could be responsible for the procollagen. Failure to demonstrate $\alpha$-actin in areas positive for procollagen would suggest that procollagen production is unlikely to be attributable to smooth muscle cells.

Sections were examined by independent observers blinded as to the identity of the specimens. Sections were graded 0 (for no procollagen seen), $1+$ (for isolated and scattered foci of procollagen), or $2+$ (for larger and contiguous procollagen deposits). Grading was applied to each specimen on the basis of the largest concentration of procollagen, regardless of the location within the specimen. After histologic scoring was complete, the code was broken and the identity of the specimens was determined. For each rat, the histologic score of the native valve was compared with that of the graft. This method provides reproducible, qualitative evaluation for procollagen presence.

\section{Results}

Typical photomicrographs of specimens stained for procollagen and $\alpha$-actin are shown in Figs. 1 and 2 . By far, the greatest concentration of procollagen was in the hinge region of the valve, where the leaflet arose from the aortic wall. Some additional procollagen was less frequently observed in the aortic wall at sites more remote from the hinge region. Procollagen was never observed in the free portion of the valve leaflets beyond the hinge region. 

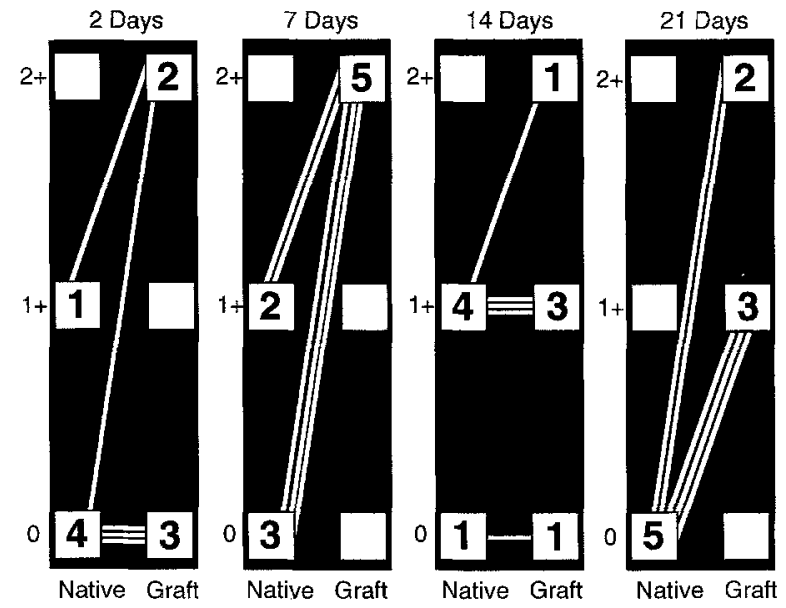

Fig. 3. Immunocytochemical procollagen score for fresh isograft group. Lines connect each aortic valve graft with its corresponding native valve.

Comparison of the two photomicrographs demonstrates that the area positive for procollagen is remote from that positive for $\alpha$-actin. This pattern of disparate areas of procollagen and $\alpha$-actin staining was observed in all specimens. The cryopreserved grafts were notable for the absence of endothelium, unlike the fresh grafts. This observation has been reported previously from this laboratory. ${ }^{4}$

The density of procollagen staining in the grafts equaled or exceeded that in the corresponding native aortic root in all cases. The procollagen grading was not substantially different at any of the time points studied. No differences in concentration or pattern of procollagen presence were observed in fresh grafts compared with cryopreserved grafts or in syngeneic compared with allogeneic grafts. It is not possible from these observations to determine whether the presence or location of procollagen suggests an adaptive or a maladaptive process. A schematic representation of the procollagen grading is displayed in Figs. 3 through 6.

\section{Discussion}

Despite the long-standing and increasingly popular use of aortic allografts for the treatment of congenital and acquired heart disease, many basic questions remain regarding the cellular biology of these tissues. Whether the cells constituting the graft remain alive, whether they are capable of normal cellular processes, and whether they are affected by techniques of harvest, sterilization, and storage have been vigorously debated. Establishing

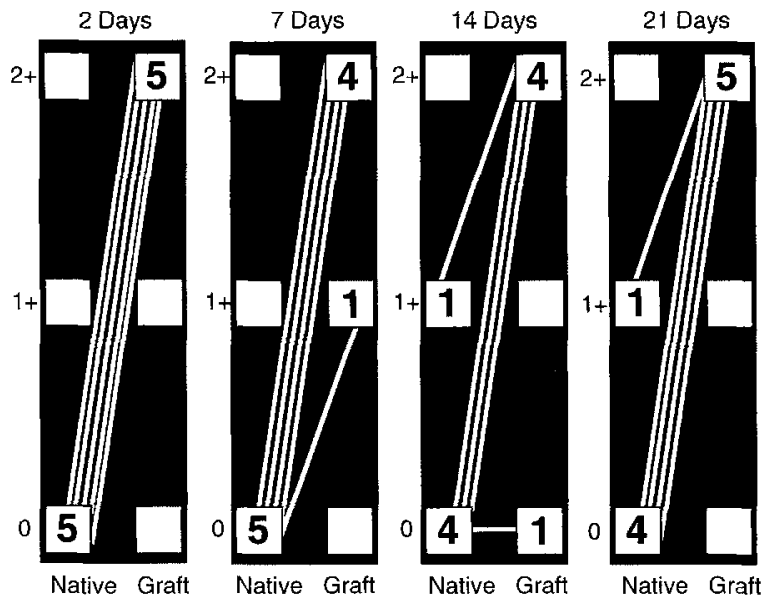

Fig. 4. Immunocytochemical procollagen score for fresh allograft group. Lines connect each aortic valve graft with its corresponding native valve.

the answers to some of these questions on the basis of clinical evidence is difficult at best. Some of the difficulties in analyzing the reasons for graft dysfunction are that the longevity of these grafts is generally good, and even grafts that fail may not be subjected to careful examination. Furthermore, "graft failure" often results not from tissue degeneration but instead is a consequence of surgeon error, the use of other materials that predispose to graft obstruction, or simple growth of the patient's heart beyond the size appropriate for the graft. ${ }^{11-13}$ Accordingly, experimental models of allograft valve implantation may provide a critical link between basic cell biology and clinical inferences.

The use of the rat heterotopic graft model has provided useful information regarding the immunologic status and endothelial cell viability of aortic valve grafts. This experimental preparation exposes the graft to the same arterial oxygen content and $\mathrm{pH}$ that an orthotopic graft would have. Certain mechanical properties, such as shear stress, pulsatility of pressure, and transvalvular gradients may be similar but are almost certainly not identical. Because procollagen synthesis may be regulated in part by these mechanical forces, ${ }^{7}$ it is noteworthy that the experimental model in this study used a competent valve graft. Unlike previous studies from this laboratory, in which one leaflet of the valve was intentionally made incompetent to reduce the frequency of thrombosis, ${ }^{2-4}$ in this investigation the valve was implanted to make it possible for all leaflets to be mobile. Whether mobility and coaptation in fact 

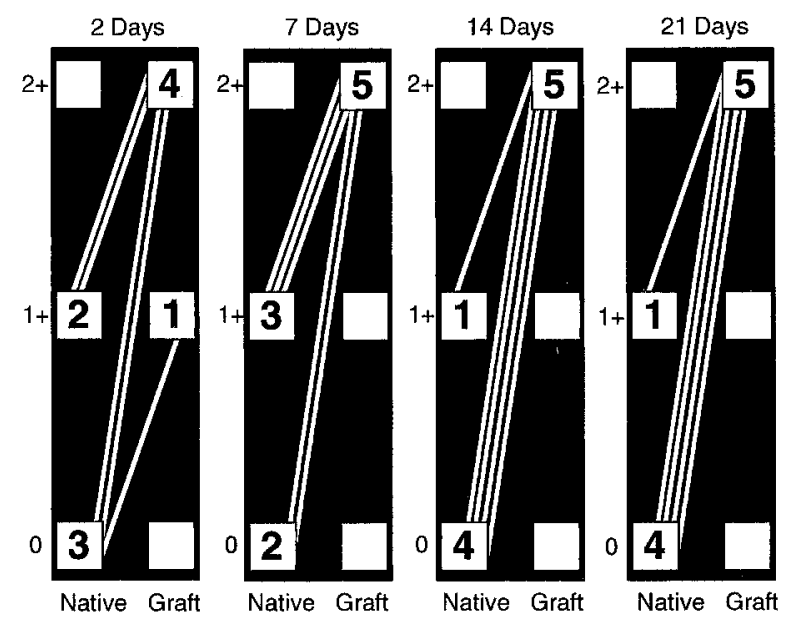

Fig. 5. Immunocytochemical procollagen score for cryopreserved isograft group. Lines connect each aortic valve graft with its corresponding native valve.

occurred is not known. Type I collagen, the type examined in this study and the most abundant procollagen subtype in the heart, is less affected by mechanical stimulation than is type III collagen, the second most abundant collagen type in the heart. ${ }^{14}$

Collagen is the predominant component of the extracellular matrix of the heart. Type I collagen does not arise from cardiac myocytes, but rather is produced principally by the fibroblasts. ${ }^{15}$ Collagen biogenesis does not occur intracellularly. Because collagen molecules spontaneously precipitate in a normal physiologic milieu, synthesis of the unaltered molecule would be incompatible with transport and assembly into fibrils. ${ }^{16}$ Instead, collagen is synthesized and secreted as procollagen, a precursor molecule that is soluble in intracellular and extracellular fluids. This permits transfer of procollagen to sites where the amino and carboxy terminal propeptides are removed and collagen polymerizes in a helical secondary structure. ${ }^{17}$ After secretion of procollagen, the propeptides are rapidly proteolyzed. ${ }^{18}$ Antibodies to the propeptide therefore identify sites of active collagen synthesis, whether associated with rapid growth, trauma, or inflammatory fibrosis, but do not localize to quiescent locations of past collagen assembly. ${ }^{19}$ Because procollagen was demonstrated in aortic valve grafts at all intervals studied from 2 to 21 days after implantation, it is concluded that this synthetic activity is occurring in the earliest postoperative period and continues for a prolonged period thereafter.

Like the fibroblasts, vascular smooth muscle cells are capable of procollagen production. The obser-

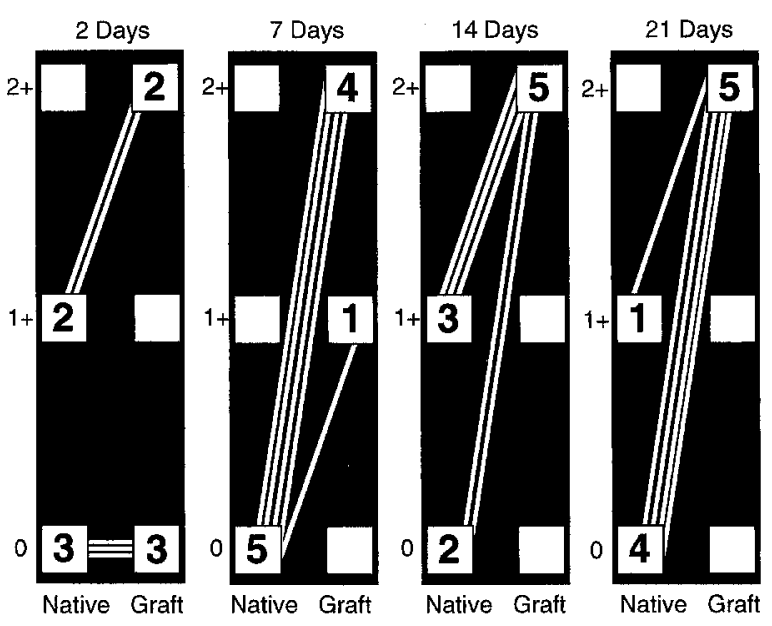

Fig. 6. Immunocytochemical procollagen score for cryopreserved allograft group. Lines connect each aortic valve graft with its corresponding native valve.

vation that $\alpha$-actin staining did not coincide with procollagen staining indicates that vascular smooth muscle is not the source of the procollagen observed in the aortic valve grafts. This indirectly supports fibroblasts as the cell population responsible for procollagen. This cannot be directly confirmed because of the lack of a specific marker for fibroblasts. It remains a possibility that vascular smooth muscle cells dedifferentiate into multipotential cells that synthesize procollagen while losing the ability to synthesize $\alpha$-actin. Another intriguing possibility was suggested by the studies of Messier and colleagues. ${ }^{20}$ These investigators defined a population of aortic valve leaflet interstitial cells that exhibited properties of both fibroblasts and smooth muscle cells. They proposed designating these cells as "myofibroblasts" to emphasize their unique properties and raised the hypothesis that those properties contribute to leaflet durability. ${ }^{20}$

It seems reasonable that the observation of procollagen in the aortic valve graft can be taken as evidence for structural repair. This hypothesis would be consistent with the understanding that the hinge point of the valve leaflets, where procollagen was found in greatest abundance, is the site of maximum tissue stress and has the greatest requirements for repair. In the embryonic heart, the cardiac valve anlage demonstrates maximum collagen distribution in the valve ring area and arterial wall with little or no collagen in the commissural areas. ${ }^{21}$ Thus the pattern of procollagen production in the valve grafts suggests a pattern similar to that seen in the devel- 
oping valve. It may be speculated that the grafted aortic valve responds to surgical injury by means of a process reminiscent of early development.

On the other hand, it is possible that procollagen production is not directly associated with repair but in fact presages a fibrous dysplasia. This may in turn lead to restriction of leaflet mobility, calcification, and obstruction to flow. At this time, it is not possible from histologic examination to distinguish between procollagen production leading to tissue support and maintenance of structural integrity and that which results in dystrophic changes and impaired valve function. Evidence that collagen is increased in pathologic valves has been demonstrated by staining with ruthenium red, which delineates mucopolysaccharide in the center of collagen fibrils and surrounding the fibrils. ${ }^{22}$ Procollagen production is also upregulated in atherosclerotic plaques. ${ }^{8}$ At least in atherosclerotic plaque, procollagen production is not found to be upregulated in areas of active cellular proliferation. ${ }^{9}$

The findings of this study suggest that fibroblast viability is retained in this model of aortic valve grafts and that capacity for synthesis of an important structural protein persists. Staining for procollagen in the graft was equal to or greater than that in the corresponding native valve, suggesting that procollagen synthesis is increased over baseline levels. This increase may be a response to surgical trauma, ischemia and reperfusion, growth factor elaboration, or other causes. The similarities in the fresh and cryopreserved grafts provide evidence that cryopreservation is compatible with intact fibroblast function. Finally, the similarities in the syngeneic and allogeneic grafts indicate that histocompatibility differences do not affect these properties within the time frame studied.

\section{REFERENCES}

1. Yankah AC, Dreyer W, Wottge HU, Muller-Rucholtz W, Bernhard A. Kinetics of endothelial cells of preserved aortic valve allografts used for heterotopic transplantation in inbred rat strains. In: Bodnar $\mathrm{E}$, Yacoub $\mathbf{M}$, eds. Biologic and bioprosthetic valves. New York: Yorke Medical, 1986:73-84.

2. Christy JP, Lupinetti FM, Mardan AH, Thompson SA. Endothelial viability in the rat aortic wall. Ann Thorac Surg 1991;51:204-7.

3. Lupinetti FM, Christy JP, King DM, El Khatib H, Thompson SA. Immunogenicity, antigenicity, and endothelial viability of aortic valves preserved at $4^{\circ} \mathrm{C}$ in a nutrient medium. J Card Surg 1991;6:454-61.

4. Lupinetti FM, Tsai TT, Kneebone JM. Endothelial cell viability in an in vivo model of aortic allografts. Ann Thorac Surg 1993;56:237-41.

5. Lupinetti FM, Tsai TT, Kneebone JM, Bove EL. Effect of cryopreservation on the presence of endothelial cells on human valve allografts. J Thorac Cardiovasc Surg 1993;106:912-7.

6. Foellmer HG, Kawahara K; Madri JA, Furthmayr H, Timpl R, Tuderman L. A monoclonal antibody specific for the amino terminal cleavage site of procollagen type 1. Eur J Biochem 1983;134:183-9.

7. Sinning AR, Lepera RC, Markwald RR. Initial expression of type I procollagen in chick cardiac mesenchyme is dependent upon myocardial stimulation. Dev Biol 1988;130:167-74.

8. Rekhter MD, Zhang K, Narayanan AS, Phan S, Schork MA, Gordon D. Type I collagen gene expression in human atherosclerosis: localization to specific plaque regions. Am J Pathol 1993;143:1634-48.

9. Rekhter MD, Gordon D. Cell proliferation and collagen synthesis are two independent events in human atherosclerotic plaques. J Vasc Res 1994;31:280-6.

10. Skalli O, Ropraz P, Trzeciak A, Benzonana G, Gillessen D, Gabbiani G. A monclonal antibody against alpha-smooth muscle actin: a new probe for smooth muscle differentiation. J Cell Biol 1986;103:2787-96.

11. Kobayashi J, Backer CL, Zales VR, Crawford SE, Muster AJ, Mavroudis C. Failure of the Hemashield extension in right ventricle-to-pulmonary artery conduits. Ann Thorac Surg 1993;56:277-81.

12. Hawkins JA, Bailey WW, Dillon T, Schwartz DC. Midterm results with cryopreserved allograft valved conduits from the right ventricle to the pulmonary arteries. J Thorac Cardiovasc Surg 1992;104:910-6.

13. Levine JC, Mayer JE Jr, Keane JF, Spevak PJ, Sanders SP. Anastomotic pseudoaneurysm of the ventricle after homograft placement in children. Ann Thorac Surg 1995;59:60-6.

14. Carver W, Nagpal ML, Nachtigal M, Borg TK, Terracio L. Collagen expression in mechanically stimulated cardiac fibroblasts. Circ Res 1991;69:116-22.

15. Eghbali M, Czaja MJ, Zeydel M, et al. Collagen chain mRNAs in isolated heart cells from young and adult rats. $\mathbf{J}$ Mol Cell Cardiol 1988;20:267-76.

16. Minor RR. Collagen metabolism: a comparison of diseases of collagen and disease affecting collagen. Am J Pathol 1980; 98:227-78.

17. Marchi F, Leblond CP. Collagen biogenesis and assembly into fibrils as shown by ultrastructural and $3 \mathrm{H}$-proline radioautographic studies on the fibroblasts of the rat foot pad. Am J Anat 1983;168:167-97.

18. Fessler JH, Fessler LI. Biosynthesis of procollagen. Annu Rev Biochem 1978;47:129-62.

19. McDonald JA, Broekelmann TJ, Matheke ML, Crouch E, Koo M, Kuhn C III. A monoclonal antibody to the carboxyterminal domain of procollagen type I visualizes collagensynthesizing fibroblasts. J Clin Invest 1986;78:1237-44.

20. Messier RH Jr, Bass BL, Aly HM, et al. Dual structural and functional phenotypes of the porcine aortic valve interstitial population: characteristics of the leaflet myofibroblast. J Surg Res 1994;57:1-21.

21. Garcia-Martinez V, Sanchez-Quintana D, Hurle JM. Histogenesis of the semilunar valves: an immunohistochemical analysis of tenascin and type-I collagen distribution in developing chick heart valves. Cell Tissue Res 1990;259:299-304.

22. Nakao K, Bashey RI. Fine structure of collagen fibrils as revealed by ruthenium red. Exp Mol Pathol 1972:17:6-13. 